\title{
Study of Amplifier Operating Point to Improve Phonocardiogram Signal Representation in a Custom-made Electronic Stethoscope
}

\author{
Juli Astono, Heru Kuswanto, Sumarna Sumarna, Wipsar Sunu Brams Dwandaru \\ Physics Education Department, Faculty of Mathematics and Natural Sciences, Universitas Negeri \\ Yogyakarta, Jl. Colombo No. 1, Karangmalang Complex, Yogyakarta, 55281, Indonesia
}

\begin{abstract}
The performance of a custom-made electronic stethoscope needs to be improved through a study on the operating point of the amplifier. The aim of this study is to analyse the composition and content of the frequency components contained in the phonocardiogram signal by spectral extraction. The output is a voltage signal that represents the heart rate. The operating point of the amplifier is varied. The signal is recorded on a laptop and analysed. The results show that the operating point produces a balanced swing signal. Hence, the amplifier's operating point does not affect the component spectrum in the recorded signal.
\end{abstract}

Keywords - amplifier's operating point, phonocardiogram signal, custom-made electronic stethoscope.

\section{Introduction}

Phonocardiogram (PCG) signals include biomedical signals originating from the heart in the human body [1].

DOI: 10.18421/TEM111-21

https://doi.org/10.18421/TEM111-21

Corresponding author: Wipsar Sunu Brams Dwandaru, Physics Education Department, Faculty of Mathematics and Natural Sciences, Universitas Negeri Yogyakarta, Karangmalang Complex, Yogyakarta, 55281, Indonesia.

Email: wipsarian@uny.ac.id

Received: 04 September 2021.

Revised: 20 January 2022.

Accepted: 26 January 2022.

Published: 28 February 2022.

(c))BY-NC-ND (C) 2022 Juli Astono et al; published by UIKTEN. This work is licensed under the Creative Commons Attribution-NonCommercial-NoDerivs 4.0 License.

The article is published with Open Access at https://www.temjournal.com/
PCG signals contain very important information regarding a person's health status. PCG signals first have to be analyzed before a person's health condition can be identified and described. Therefore, a representative PCG signal can provide representative health status information.

Cardiac abnormalities have been investigated in various ways, including using the electrocardiogram (ECG) test, which has been used as a non-invasive method of investigation [2]. The ECG test varies if the heart rate signal from the PCG shows abnormalities so PCG is also used to increase the efficiency and accuracy of heart diagnosis. Study of abnormal heart rate (rhythm) has important clinical meaning. PCG signals are also used to diagnose cardiac abnormalities [3]. The heart consists of two parts related to pumping, i.e.: mechanical and electrical parts. The mechanical function of the heart is regulated by the electrical system in it. ECG shows electrical activity and PCG shows mechanical activity (valve opening and closing) of the human heart so that there is an important relationship between ECG and PCG signals [4].

Extracting information from within the PCG requires a mechanism because of the PCG signal's unique feature to provide data in the diagnostic process and the extracted profile has to be optimal for accurate detection of heart pathological conditions. PCG signal is a weak acoustic signal, but has significant profile parameters such as entropy, energy, standard deviation, variance, and others. Profile extraction can be conducted in various ways such as statistical, fractional Fourier transform [5], and wavelet transform [6]. However, when physical spectral analysis is desired, the most optimal method is spectral extraction [7].

Modern technology provides highly efficient tools for evaluating heart rate-related information that is not available in traditional tools such as stethoscopes. One of the most widely used methods for listening and tracking the heartbeat is recording it with a special device. PCG signal can be used to distinguish 
normal and abnormal heart rate [8]. Furthermore, the development in computer technology has made it possible to improve the quality of PCG signals that are digitally recorded, managed, and manipulated to test frequency content. In addition, the development of new techniques for digital signal processing such as pattern recognition and time-frequency analysis has improved PCG signal analysis and made it a noninvasive technique for diagnosing cardiac activity [9]. Heart murmurs provide the first indication of these morphological changes and are primarily found on auscultation throughout medical care. PCG morphological changes come from the heartbeat in the form of changes in shape, duration, and amplitude.

Extraction of the frequency components contained in the heartbeat signal can be done by spectral extraction compiled using the MATLAB program. The signal is the result of an electro-acoustic recording in the form of a PCG signal. In this case, a homemade electronic stethoscope has been produced. This device was created to improve the shortcomings of conventional acoustic stethoscopes [10]. This device was first designed and created in [11]. Furthermore, the PCG signal from this device is improved in [10]. Especially in this study, the component spectrum of the resulting signal is determined. The difference in spectrum due to variations in the position of the operating point of the electronic stethoscope amplifier proves that this part affects the representation of the components contained in the heartbeat signal. Thus, the composition and content of the frequency components contained in the PCG signal is analyzed by means of spectral extraction.

\section{Experimental}

The object of this study was the custom-made human heart rate detection device. The analysis software used was the spectral extraction. The detection device was designed and assembled by utilizing the basic components of a stethoscope, i.e.: a chest piece, an op-amp, a transistor, a resistor, a mic condenser, and a capacitor. Additional equipment used was a laptop and an oscilloscope. The software used in this study was SoundForge 10, MATLAB, and spectral extraction program. An illustration of the custom-made stethoscope can be observed in Figure 1. Moreover, the electronic circuit of the stethoscope can be observed in Figure 2.

The data in this study were obtained through observation, namely by recording the regenerated heart rate by varying the position of the operating point of the electronic amplifier, each of which produces a signal that was stored in the form of a wav file. Each signal was then processed using analysis software with spectral extraction to obtain the frequency spectrum (composition and component contents).
The data were analyzed by quantitative and comparative descriptive methods, namely describing the composition of the frequency and amplitude of each spectrum, which has been represented in the form of numbers. The comparison process used regenerated heart rate recordings where the position of the amplifier's operating point varies for the same source.

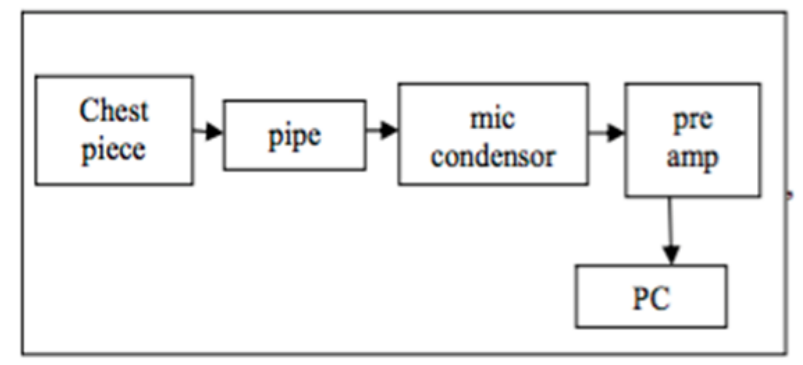

Figure 1. The custom-made electronic stethoscope

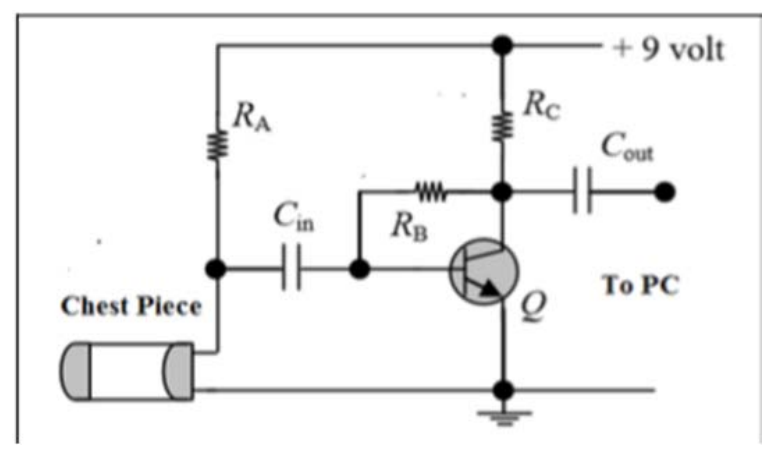

Figure 2. The electronic circuit of the custom-made electronic stethoscope

\section{Results and Discussion}

This study utilizes a prototype of human heart rate detection device, i.e.: a custom-made electronic stethoscope based on the existing electro-acoustic principle to record the regenerated heartbeat. The use of regenerated heartbeats is intended to ensure consistency in the composition and frequency content of the signal source. The output of this device is a voltage signal that represents the heartbeat. The electronic amplifier part of the prototype is varied in the position of its operating point. The signal is then recorded on a laptop using SoundForge 10 software in the form of a .wav format. The signal from this recording is then analyzed for the composition and content of its frequency components using spectral extraction. The results of the analysis are presented in Figure 3.

If we pay close attention to the spectral analysis results in Figure 3, where each image comes from different amplifier operating points, it turns out that in each bin the corresponding frequency in the range of $0 \mathrm{~Hz}$ to $200 \mathrm{~Hz}$ has the same amplitude intensity. Furthermore, the comparison of the spectral of the seven images is summarized in Figure 4, which shows that the spectrum content has not changed. 
TEM Journal. Volume 11, Issue 1, pages 174-178, ISSN 2217-8309, DOI: 10.18421/TEM111-21, February 2022.
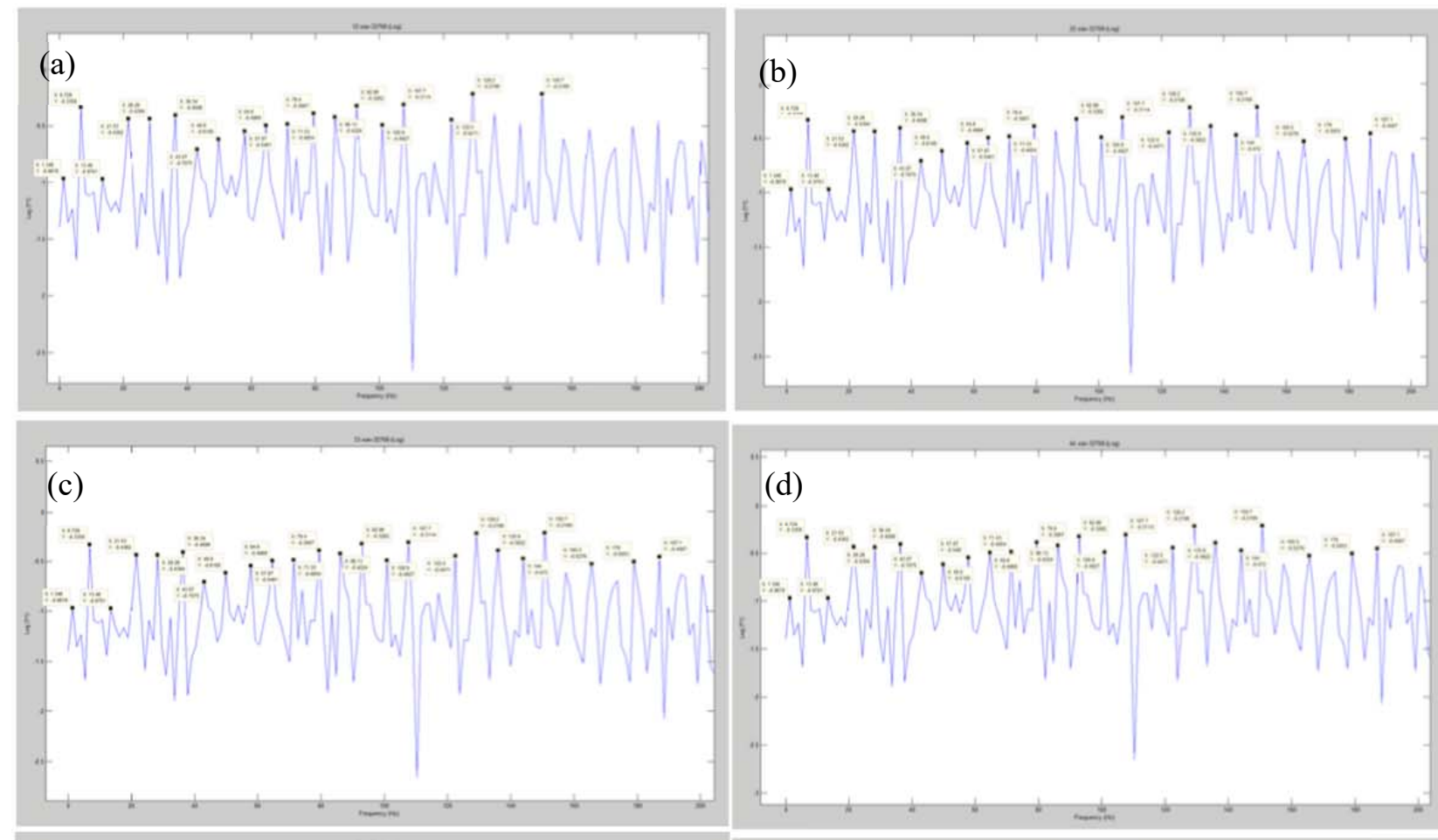

(d)

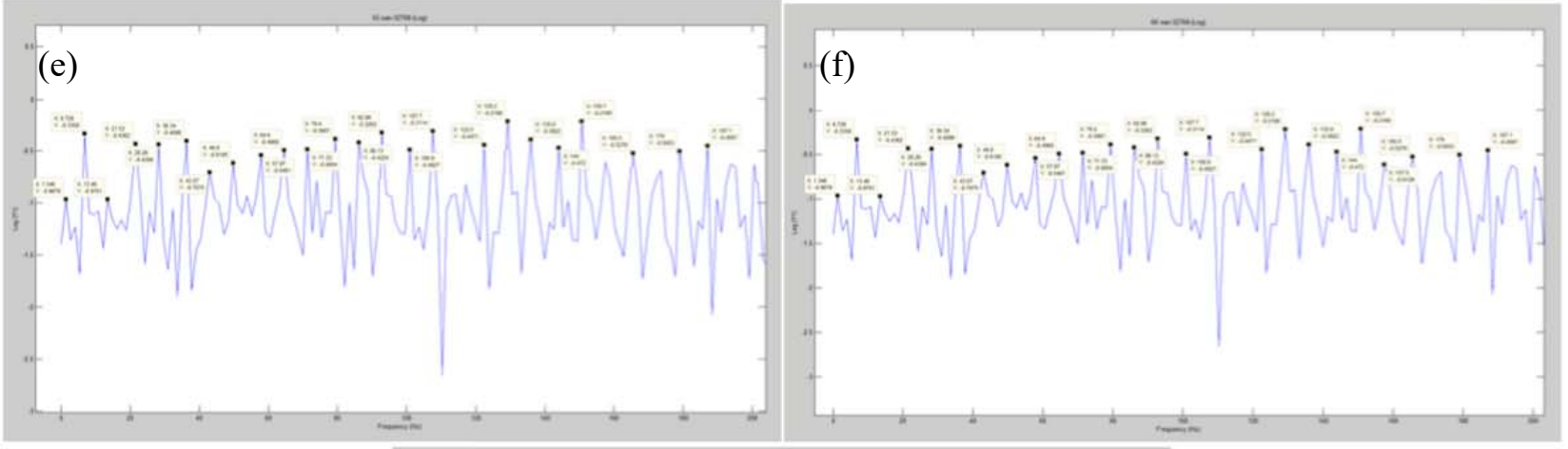

(g)

Figure 3. PCG spectral at operating points of 12 (a); 22(b); 33(c); 44(d); 55(e); 66(f); and 77(g)

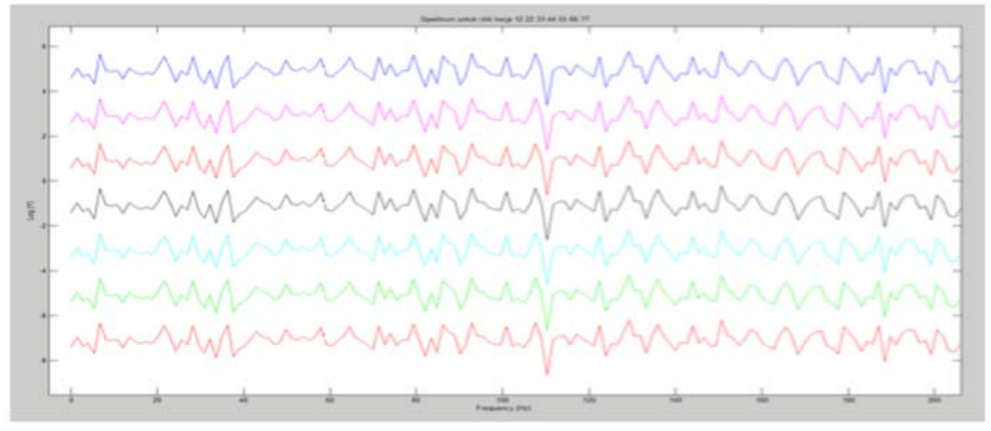

Figure 4. Comparison of the PCG spectral from operating points of 12, 22, 33, 44, 55, 66, and 77 from top to bottom, respectively 
This situation occurs because the regenerating heartbeat signals, both input and output do not experience amplitude cuts (illustration given in Figure 5), meaning that both the current swing and the output voltage swing are still in balance. The shape of a balanced swing signal can be seen in Figure 6, while the shape of an unbalanced (truncated) signal swing is illustrated in Figure 7.

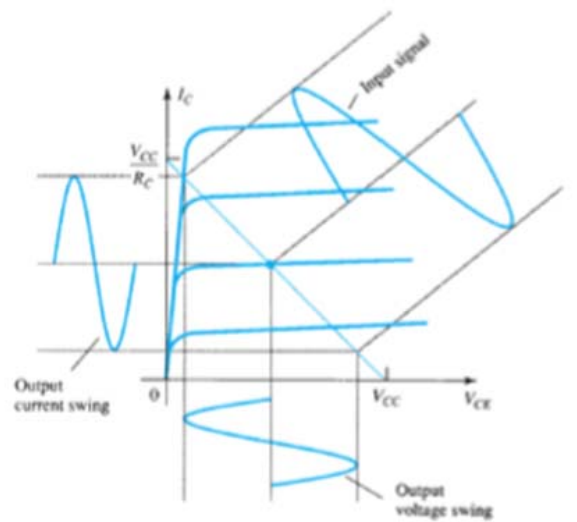

Figure 5. An illustration of the operating point position on the $\left(V_{C E}, V_{C C} / R_{C}\right)$ coordinate

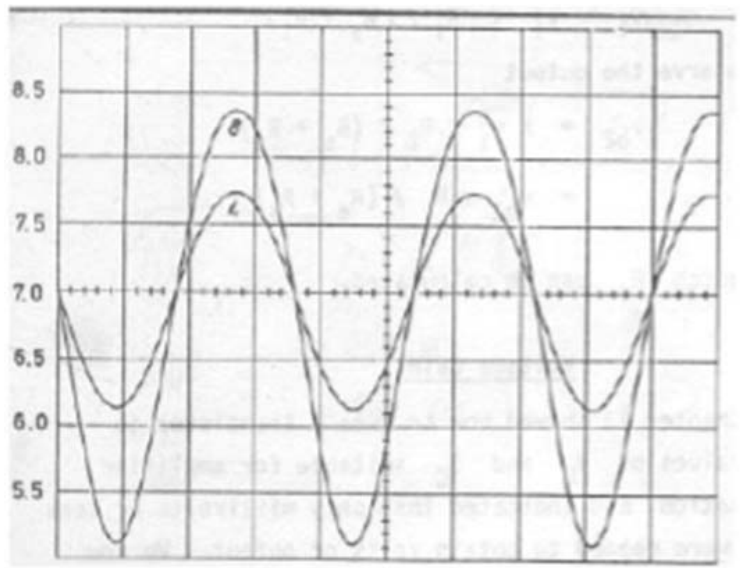

Figure 6. A balanced signal swing form

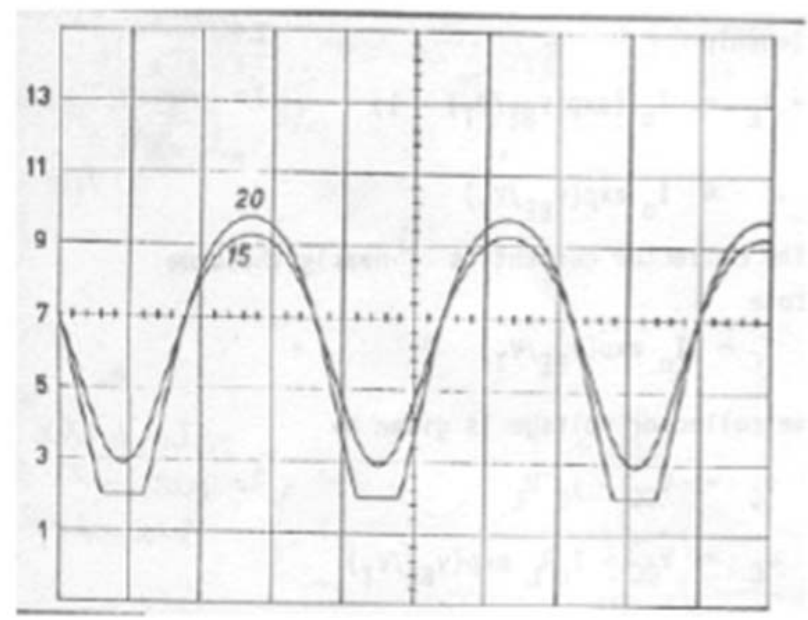

Figure 7. An unbalanced signal swing form

\section{Conclusions}

As long as the positioning of the operating points ensures that the processed swing signal is balanced (no part of the output amplitude is cut off), then the operating point of the amplifier (electronic circuit part) of the custom-made electronic stethoscope does not affect the information content or component spectrum contained in the recorded signal. Based on the results of this study, it can be suggested that when designing and manufacturing an electronic stethoscope, it is ensured that the operating point of the electronic circuit, especially the amplifier part, is in a position that allows a balanced signal swing to occur, meaning that there is no signal amplitude cut. Thus, the position of the operating point is not a unique and crucial issue. The position of the operating point can be placed along the load line as long as the swing signal is guaranteed to be balanced.

\section{Acknowledgements}

The authors would like to thank the Faculty of Mathematics and Natural Sciences, Universitas Negeri Yogyakarta for funding this study through the Research Group Grant of 2020 - 2021 with contract number: B/171/UN34.13/PM.01.01/2020 and project title "A Study on the Custom-made Electronic Stethoscope".

\section{References}

[1]. Tiwari, S., Jain, A., Sharma, A. K., \& Almustafa, K. M. (2021). Phonocardiogram signal based multi-class cardiac diagnostic decision support system. IEEE Access, 9, 110710-110722.

[2]. Boonstra, M. J., Roudijk, R. W., Van Dam, P. M., Van Der Heijden, J. F., Asselbergs, F. W., \& Loh, P. (2021). In-vivo endocardial and epicardial quantitative comparison of multi-wave based noninvasive inverse electrocardiography. EP Europace, 23(Supplement_3), euab116-341.

[3]. Zeng, W., Lin, Z., Yuan, C., Wang, Q., Liu, F., \& Wang, Y. (2021). Detection of heart valve disorders from PCG signals using TQWT, FA-MVEMD, Shannon energy envelope and deterministic learning. Artificial Intelligence Review, 1-38.

[4]. Ajitkumar Singh, S., Ashinikumar Singh, S., Dinita Devi, N., \& Majumder, S. (2021). Heart Abnormality Classification Using PCG and ECG Recordings. Computación y Sistemas, 25(2), 381-391.

[5]. Abduh, Z., Nehary, E. A., Wahed, M. A., \& Kadah, Y. M. (2020). Classification of heart sounds using fractional fourier transform based mel-frequency spectral coefficients and traditional classifiers. Biomedical Signal Processing and Control, 57, 101788.

[6]. Dhar, P., Dutta, S., \& Mukherjee, V. (2021). Crosswavelet assisted convolution neural network (AlexNet) approach for phonocardiogram signals classification. Biomedical Signal Processing and Control, 63, 102142. 
[7]. Berraih, S. A., Debbal, S. M. E. A., Yettou, \& N. E. B. (2021). Severity cardiac analysis using the higherorder spectra. Applied Mathematics and Computation, 409, 126389.

[8]. Zannat, F., Khan, M. M., \& Al Sohad, S. (2021, April). Automated System for Features Extraction From PCG Signal. In 2021 5th International Conference on Computing Methodologies and Communication (ICCMC) (pp. 1-7). IEEE.

[9]. Banerjee, R., Choudhury, A. D., Datta, S., Pal, A., \& Mandana, K. M. (2017). Non invasive detection of coronary artery disease using PCG and PPG. In eHealth $360^{\circ}$ (pp. 241-252). Springer, Cham.
[10]. Sumarna, S., Astono, J., Purwanto, A., \& Agustika, D. K. (2017). The improvement of phonocardiograph signal (PCG) representation through the electronic stethoscope. Proceeding of the Electrical Engineering Computer Science and Informatics, 4(1), 145-149.

[11]. Sumarna, S., Purwanto, A., \& Agustika, D. K. (2016). Frequency Component Extraction of Heartbeat Cues With Short Time Fourier Transform (STFT). Jurnal Sains Dasar, 5(1), 1-6. 